\title{
Prevalência de transtorno depressivo maior em centro de referência no tratamento de hipertensão arterial
}

\author{
Prevalence of major depressive disorders in a reference center for the treatment of \\ hypertension
}

\author{
Geraldo Francisco do Amaral ${ }^{1}$, Paulo César Brandão Veiga Jardim², Marco Antonio Alves Brasil ${ }^{3}$, \\ Ana Luiza Lima Souza ${ }^{4}$, Helberte Fernandes Freitas ${ }^{5}$, Larissa Mayumi Taniguchi ${ }^{5}$, Aline Ferreira \\ Bandeira de Melo ${ }^{5}$, Carolina Nazeozeno Ribeiro ${ }^{5}$
}

${ }^{1}$ Doutor em Ciências da Saúde, Universidade de Brasília (UnB), Brasília, DF, e Universidade Federal de Goiás (UFG), Goiânia, GO. Professor adjunto, Departamento de Saúde Mental e Medicina Legal, Faculdade de Medicina, UFG. ${ }^{2}$ Doutor em Cardiologia, Universidade de São Paulo (USP), São Paulo, SP. Professor adjunto, Departamento de Clínica Médica, Faculdade de Medicina, UFG. ${ }^{3}$ Doutor em Psiquiatria, Universidade Federal do Rio de Janeiro (UFRJ), Rio de Janeiro, RJ. Chefe, Serviço de Psiquiatria, Hospital Universitário, UFRJ. ${ }^{4}$ Doutora em Saúde Pública/ Epidemiologia, USP. Professora adjunta, Faculdade de Enfermagem, UFG. Vice-coordenadora, Liga de Hipertensão Arterial, UFG. ${ }^{5}$ Médico residente.

Trabalho realizado na Liga de Hipertensão Arterial e no Departamento de Saúde Mental e Medicina Legal, Faculdade de Medicina, UFG, e no Departamento de Psiquiatria e Medicina Legal, UFRJ.

\section{Resumo}

Objetivo: Investigar a prevalência de transtorno depressivo maior em pacientes hipertensos matriculados em um centro de referência universitário para tratamento de hipertensão arterial e fatores de risco cardiovascular.

Método: Estudo transversal, descritivo, em amostra aleatória representativa, obtida de forma sistemática, de pacientes em atendimento contínuo na Liga de Hipertensão Arterial da Universidade Federal de Goiás. Aplicou-se o Inventário de Depressão de Beck para rastreamento de sintomas depressivos e a Entrevista Estruturada para o Manual de Diagnóstico e Estatística das Perturbações Mentais - Transtornos do Eixo I para avaliação diagnóstica de transtorno depressivo maior. Foram constituídos um grupo com pacientes portadores de depressão maior, denominado grupo-estudo, e um grupo com pacientes não-portadores de depressão maior, denominado grupo-controle. Avaliou-se variáveis sociodemográficas, pressão arterial e bioquímica sangüínea no momento da coleta de dados.

Resultados: Foram entrevistados 285 pacientes, tendo sido encontrada prevalência de $20 \%$ de depressão maior na população investigada. A idade média foi significativamente menor para o grupo-estudo, com predomínio do sexo feminino. A prática de atividade física regular foi também significativamente menor entre os pacientes do grupo-estudo, que também apresentaram valores mais elevados de pressão arterial diastólica e de colesterolemia.

Conclusões: Foi encontrada uma prevalência de transtorno depressivo maior em pacientes hipertensos superior àquela encontrada na população geral. Isso aponta para uma necessidade de maior atenção ao diagnóstico dos transtornos depressivos em pacientes hipertensos em atendimento primário e ambulatorial.

Descritores: Prevalência, sintomas depressivos, depressão maior, hipertensão arterial, comorbidade.

\begin{abstract}
Objective: To investigate the prevalence of major depression disorders in hypertensive patients enrolled in a university reference center for treatment of hypertension and other cardiovascular risk factors.
\end{abstract}


Methods: Cross-sectional, descriptive study of a representative randomized sample of patients, obtained according to a systematic protocol, among individuals enrolled for continuous treatment at the Hypertension League of Universidade Federal de Goiás. The Beck Depression Inventory was administered to detect depressive symptoms, and the Structured Clinical Interview for the Diagnostic and Statistical Manual of Mental Disorders for diagnostic classification of major depressive disorders. Two groups were formed, one with patients with major depressive disorder, called study group, and another with patients without major depression, called control group. Sociodemographic variables, blood pressure and plasma biochemistry were evaluated at the time of data collection.

Results: A total of 285 patients were evaluated and results indicated a $20 \%$ prevalence of major depression in the population included in the study. Mean age was significantly lower for the study group, in which female patients were predominant. Regular physical activity was significantly lower among patients in the study group, and higher diastolic blood pressure values as well as cholesterolemia were also found in this group.

Conclusions: These results show a higher prevalence of major depressive disorder among these hypertensive patients, compared with the general population. More attention should be paid to establishing an adequate diagnosis for depressive disorders in hypertensive patients, both in primary care facilities and in outpatient clinics.

Keywords: Prevalence, depressive symptoms, major depression, hypertension, comorbidity.

\section{Introdução}

Nas últimas décadas, investigações clínicas e revisões têm sugerido a associação entre transtornos depressivos (TD) e hipertensão arterial (HA), com alguns estudos mostrando consistência quanto às interações entre essas enfermidades, tanto no aspecto psicossocial quanto genético, e notadamente quanto ao fato de que a depressão pode ser um fator de risco independente para $\mathrm{HA}$, tanto em homens quanto em mulheres ${ }^{1-12}$.

O transtorno depressivo maior (TDM) é considerado uma das mais prevalentes e graves doenças entre todas as enfermidades médicas. Essa afirmativa se justifica porque o TDM se apresenta com episódios de longa duração e altas taxas de cronicidade e de recorrência, levando a perdas profissionais, prejuízo físico e psíquico, além de considerável morbimortalidade por suicídio ou por associação com outras doenças ${ }^{13}$. A prevalência descrita para toda a vida do TDM é de $16 \%$, e a incidência para $\mathrm{o}$ ano anterior foi de $6,6 \%{ }^{14}$. Em atendimento primário, estudos indicam a prevalência de 4,8 a $13 \%$ de pacientes com sintomas depressivos ${ }^{15,16}$, sendo que somente a metade destes é identificada, e menos de $25 \%$ recebem tratamento minimamente adequado $\mathrm{o}^{14,17}$.

TDM é descrito como um fator de risco independente para HA, principalmente se acontecem episódios recorrentes ou mesmo um longo tempo de desenvolvimento da doença ${ }^{18}$. No entanto, outras variáveis podem estar presentes, como aquelas relacionadas ao estilo de vida (uso de álcool, tabagismo, obesidade e sedentarismo) ${ }^{19}$. Variáveis sociodemográficas e situações permanentes de estresse são também importantes desencadeadores tanto de HA quanto de TDM, o que pode ser observado em comunidades com baixo nível educacional e status ocupacional e com elevadas taxas de desajustes familiares e de criminalidade?

A HA é a mais comum das doenças crônicas em atendimento ambulatorial e a maior causa de morbimortalidade entre adultos. Sua prevalência varia de acordo com regiões, podendo ser encontrados valores de $4 \%$ na China a mais de $30 \%$ nos EUA. No Brasil, as taxas variam de 22 a $43 \%$, dependendo da região do país ${ }^{20,21}$. Parte considerável dos pacientes hipertensos apresenta comorbidade com diabetes, obesidade e dislipidemia, além de outros fatores de risco, como sedentarismo e tabagismo ${ }^{20,21}$.

Questões socioculturais, ambientais, baixo nível de escolaridade e renda familiar, além de ingesta de sódio, têm influência no aumento dos valores pressóricos ${ }^{22,23}$. A HA primária é encontrada em $95 \%$ dos portadores de HA, sendo que apenas $5 \%$ dos indivíduos têm o diagnóstico de hipertensão secundária ${ }^{24}$.

As V Diretrizes Brasileiras de Hipertensão Arterial ${ }^{24}$ e o The Seventh Report of the Joint National Committee on Prevention, Detection, Evaluation and Treatment of High Blood Pressure ${ }^{25}$ definem como hipertensos indivíduos com pressão arterial igual ou maior que $140 \mathrm{x}$ $90 \mathrm{~mm} / \mathrm{Hg}$.

A prevalência de TD em população de hipertensos tem sido relatada entre 18 e $37 \%$, sendo pequeno o número de estudos que fazem essa referência ${ }^{10-12}$.

Em trabalho realizado por Fuller ${ }^{12}$, foi encontrada prevalência de $28 \%$ de hipertensão entre pacientes deprimidos em tratamento ambulatorial. Segundo esse autor, as hipóteses etiológicas para essa associação poderiam ser assim sumarizadas: 1 - um fator fisiológico comum a ambas as enfermidades; 2 - depressão como resultado de efeito adverso de medicação antihipertensiva; 3 - depressão secundária a uma doença crônica (no caso, a HA); 4 - depressão como resultado de tratamento para HA, onde a redução da pressão arterial poderia ser causa de uma insuficiência cerebral em idosos; e 5 - a associação entre essas enfermidades ser apenas coincidente.

Simonsick et al. ${ }^{10}$ sugerem que, em hipertensos, a depressão está associada a declínio da saúde, sem que, todavia, possam determinar se os sintomas depressivos são uma potencial causa ou conseqüência das complicações. Da mesma forma, Cohen et al. ${ }^{25}$ encontraram pacientes hipertensos deprimidos, 
apresentando maior taxa de infarto agudo do miocárdio quando comparados com aqueles não-deprimidos.

Autores têm sugerido que as comorbidades entre TD e HA devem ser verificadas a partir do primeiro atendimento, considerando que essa atitude pode beneficiar o paciente de forma importante ${ }^{6,7}$.

\section{Objetivos}

Investigar a prevalência de TDM em pacientes hipertensos em um centro de referência para tratamento de HA, sua correlação com sexo e idade, além de possíveis diferenças no controle da pressão arterial e bioquímica sangüínea em relação ao grupo de pacientes nãodeprimidos da amostra estudada.

\section{Método}

O estudo foi desenvolvido em parceria entre a Liga de Hipertensão Arterial da Universidade Federal de Goiás (LHA-UFG), o Departamento de Saúde Mental e Medicina Legal da Faculdade de Medicina da UFG e o Departamento de Psiquiatria e Medicina Legal da Universidade Federal do Rio de Janeiro.

\section{Desenho}

Estudo transversal, descritivo, de amostra representativa de pacientes, entre 18 e 70 anos, matriculados em um centro de referência para o tratamento da HA e de outros fatores de risco cardiovascular.

\section{Local do estudo}

A LHA-UFG é um serviço multiprofissional, localizado no Hospital das Clínicas da UFG (HC-UFG), que se dedica ao diagnóstico e tratamento de indivíduos portadores de HA e fatores de risco cardiovascular. Além da assistência oferecida ao indivíduo hipertenso, desenvolve também atividades de ensino e pesquisa, sendo campo de estágio para alunos de graduação e pós-graduação da área da saúde.

Possui, em registro ativo, 1.400 pacientes. A população da LHA é constituída de $1 / 3$ de homens e $2 / 3$ de mulheres. Os encaminhamentos são feitos pelos serviços de saúde da comunidade e de outras clínicas do HC-UFG, sendo que esse serviço é referência para a rede pública municipal e estadual.

\section{Constituição da amostra}

A amostragem foi realizada entre os pacientes matriculados na LHA-UFG localizados no arquivo ativo. Para o cálculo da amostra, foram excluídos 302 indivíduos com idade superior a 70 anos e 47 que participavam de outros estudos clínicos, sendo considerado um total de 1.051 pacientes para cálculo final da população a ser estudada.

Estimando-se a prevalência de depressão em hipertensos entre 18 e $37 \%^{10-12}$, foi calculada, como representativa dessa população, para um erro padrão de $5 \%$, uma amostra de 251 pacientes (cálculo para população finita). Foram selecionados, em amostragem aleatória sistemática, 285 indivíduos, correspondendo a $13,5 \%$ a mais que o necessário para o estudo.

Foram excluídos aqueles pacientes que se recusaram a participar do estudo e/ou com dificuldade de cooperação com a entrevista por qualquer motivo.

\section{Coleta dos dados}

Os pacientes foram selecionados de novembro de 2003 a junho de 2005, entre 8 e 9 horas da manhã de segundas, terças e sextas-feiras, através dos prontuários de pacientes previamente agendados para atendimento naquele dia. $\mathrm{Na}$ rotina diária, os prontuários são organizados pelas secretárias na recepção da LHA-UFG, em conjuntos, de acordo com o tipo de atendimento e por ordem de chegada dos pacientes: primeiro conjunto - consulta médica (até 10 pacientes); segundo conjunto - consulta de enfermagem (até 10 pacientes); terceiro conjunto consulta de nutrição (até 10 pacientes).

A seleção dos pacientes foi feita a partir do último prontuário em cada conjunto, correspondendo ao último paciente dentro da rotina de agendamento do dia, iniciando sempre pelo grupo a ser atendido pelo médico, depois pela enfermagem e, finalmente, pela nutrição.

A escolha do último prontuário em cada bloco visou não interferir na rotina de atendimento do serviço. Cada paciente selecionado foi abordado antes de sua consulta agendada, retornando para a recepção e aguardando sua consulta normalmente.

Foram selecionados, por conveniência operacional, em média, três pacientes/dia. Os pacientes foram convidados, antes do atendimento de rotina, para uma entrevista com o investigador, onde era explicado o objetivo do estudo. Após a concordância e assinatura do consentimento pós-informado, foram aplicados os instrumentos para o diagnóstico de depressão e, posteriormente, colhidos os dados dos prontuários de acordo com a consulta realizada no mesmo dia.

\section{Instrumentos de avaliação utilizados para constituição dos grupos}

Todos os pacientes selecionados foram submetidos à aplicação do Beck Depression Inventory (BDI), que é uma escala sintomática de rastreamento de depressão ${ }^{26-}$ 
uma escala sintomática de rastreamento de depressão $0^{26-}$ ${ }^{28}$. Naqueles que atingiram pontuação de 16 ou mais (depressão moderada, moderada a grave e grave), foi aplicada a Entrevista Estruturada para o Manual de Diagnóstico e Estatística das Perturbações Mentais (DSM-IV) - Transtornos do Eixo I (SCID-I/P, versão 2.0 $)^{29-31}$ para confirmação e classificação diagnóstica.

A partir da aplicação do BDI, os pacientes que apresentaram sintomas depressivos com ponto de corte $\geq 16$ foram selecionados para aplicação da SCID-I/P 2.0.

Aqueles que foram classificados pela SCID-I/P 2.0 como portadores de TDM passaram a constituir o grupoestudo (GE). O grupo-controle (GC) foi então formado pelos indivíduos que não atingiram ponto de corte $(\mathrm{BDI}<$ 16) e por aqueles que, mesmo tendo atingido o ponto de corte especificado (BDI $\geq 16$ ), foram considerados como não-portadores de TDM pela SCID-I/P 2.0 (Tabela 1).

Tabela 1 - Definição dos grupos segundo escalas utilizadas

\begin{tabular}{ll}
\hline $\mathrm{BDI} \geq 16$ pontos e SCID positivo & $\mathrm{GE}$ \\
$\mathrm{BDI} \geq 16$ e SCID negativo & $\mathrm{GC}$ \\
$\mathrm{BDI}<16$ & Grupo controle $(\mathrm{GC})$ \\
\hline
\end{tabular}

$\mathrm{GC}=$ grupo-controle; GE = grupo-estudo; SCID = Entrevista Estruturada para o Manual de Diagnóstico e Estatística das Transtornos Mentais - Transtornos do Eixo I.

\section{Ponto de corte utilizado}

Com relação ao ponto de corte, Gorenstein \& Andrade $^{27}$ e Cunha $^{28}$ mostraram estudos com pequenas variações entre os escores, geralmente diferindo nas pontuações para ausência de sintomas (0-9) e depressão leve (10-17). Em amostra de 127 pacientes adultos, Spitzer et al. ${ }^{29}$ aplicaram o BDI e a SCID-III-R (Structured Clinical Interview for DSM-III-R), conseguindo definir ponto de corte de 17 para classificação do que se considera como depressão maior. Já Splinkle et al..$^{30}$ e Beck \& Steer ${ }^{26}$ consideram que um ponto de corte entre 16 e 14 para depressão leve pode ser significativo. Beck \& Steer $^{26}$ sugerem ainda que a decisão de qual ponto de corte utilizar pode ser baseada unicamente nas características da amostra.

Sprinkle et al. ${ }^{30}$, em estudo comparativo com aquele de Beck \& Steel ${ }^{26}$, aplicaram o BDI em teste e reteste e, em seguida, o módulo de depressão da SCID-I/P 2.0, identificando ponto de corte de 17 no BDI, para correlação com depressão maior.
O interesse, neste estudo, é a identificação de comorbidade depressiva que porventura possa influir na evolução do tratamento anti-hipertensivo, e, por isso, escolheu-se utilizar escores que se situassem dentro de uma média estabelecida nos trabalhos citados. Assim é que se optou por excluir, nesta pesquisa, os pacientes com pontuação entre 10 e 15 (depressão leve), uma vez que o objeto de interesse foi identificar portadores de TDM de acordo com a DSM-IV 32 .

A pontuação utilizada para o BDI foi: 0 a 9 ausência de sintomas; 10 a 15 - depressão leve; 16 a 19 - depressão moderada; 20 a 29 - depressão moderada a grave; 30 a 63 - depressão grave.

Todos os indivíduos que atingiram pontuação $\geq$ 16 para o BDI foram analisados à luz da SCID-I/P 2.0.

\section{Aspectos éticos}

O protocolo foi revisto e aprovado pelo Comitê de Ética e Pesquisa Médica Humana e Animal do HCUFG.

Ao longo das entrevistas com o BDI e SCID-I/P 2.0, os pacientes identificados como deprimidos foram encaminhados para tratamento e acompanhamento no Ambulatório de Psiquiatria do Departamento de Saúde Mental e Medicina Legal da Faculdade de Medicina/ HC-UFG. Os pacientes que apresentaram escores no BDI para depressão leve (entre 10 e 15 pontos) e aqueles com $\mathrm{BDI} \geq 16$, mas não identificados como TDM pela aplicação da SCID-I/P 2.0, foram encaminhados para avaliação psiquiátrica no Ambulatório de Psiquiatria do HC-UFG.

\section{Parâmetros avaliados}

Os indivíduos foram avaliados em relação a:

- Pressão arterial: pressões sistólica e diastólica, sendo que para efeito de análise foi considerado o segundo valor registrado na última consulta na LHAUFG;

- Índice de massa corpórea (IMC): conforme registrado no prontuário ${ }^{33}$;

- Tabagismo: presença/ausência do hábito;

- Etilismo: presença/ausência do hábito e seu quantitativo de acordo com tabela utilizada na LHA;

- Atividade física: regular, irregular ou ausente, de acordo com a classificação utilizada na LHA (ausente - sem atividade física; irregular - menos de três vezes por semana; regular - três ou mais vezes por semana);

- Nível de colesterol, triglicérides e glicemia: de acordo com resultados registrados.

Todos os dados foram colhidos nos prontuários de acordo com a consulta realizada por ocasião da seleção. 


\section{Análise estatística}

Para a criação do banco de dados, foi utilizado o programa Excel 2000, e para a realização dos testes estatísticos, o programa SPSS 13.5. Foi utilizada a análise de variância para verificar a existência de diferença entre os grupos GE e GC quanto à idade; na caracterização sociodemográfica da amostra, utilizou-se o teste qui-quadrado, e para avaliação entre grupos, foi utilizado o teste de MannWhitney ${ }^{34}$.

\section{Resultados}

Dos 285 pacientes inicialmente selecionados para aplicação do BDI, 206 (72,3\%) tiveram pontuação inferior à estipulada $(\mathrm{BDI}<16)$ (Tabela 2). A aplicação da SCID$\mathrm{I} / \mathrm{P} 2.0$ aos $79(27,7 \%)$ pacientes que atingiram o ponto de corte mínimo $(\mathrm{BDI} \geq 16)$ confirmou o diagnóstico de TDM em 57 (20,0\%) pacientes, constituindo o GE. Os 22 $(7,7 \%)$ pacientes com $\mathrm{BDI} \geq 16$ pontos, mas não confirmados como portadores de TDM pela SCID-I/P 2.0, foram agregados aos 206 pacientes já selecionados pelo BDI, passando a constituir o GC (Tabela 3).

Tabela 2 - Classificação inicial dos pacientes com sintomas depressivos e sem sintomas depressivos segundo o BDI

\begin{tabular}{llllll}
\hline \multirow{2}{*}{ Resultados } & \multicolumn{2}{l}{$\begin{array}{l}\text { Com sintomas } \\
\text { depressivos }\end{array}$} & \multicolumn{2}{l}{$\begin{array}{l}\text { Sem sintomas } \\
\text { depressivos }\end{array}$} & \multirow{2}{*}{ Total } \\
\cline { 2 - 5 } & $\mathbf{n}$ & $\mathbf{\%}$ & $\mathbf{n}$ & $\mathbf{\%}$ & \\
\hline Não-deprimidos & - & 0 & 158 & 76,7 & 158 \\
Depressão leve & - & 0 & 48 & 23,3 & 48 \\
Depressão moderada & 16 & 15,8 & - & 0 & 16 \\
Depressão moderada a grave & 38 & 49,1 & - & 0 & 38 \\
Depressão grave & 25 & 35,1 & - & 0 & 25 \\
Total & 79 & 27,7 & 206 & 72,3 & 285 \\
\hline
\end{tabular}

Tabela 3 - Características sociodemográficas da amostra em sua composição final

\begin{tabular}{lllllll}
\hline Variáveis & \multicolumn{2}{l}{ GE $(\mathbf{n}=\mathbf{5 7})$} & \multicolumn{2}{l}{ GC $(\mathbf{n}=\mathbf{2 2 8})$} & \multicolumn{2}{c}{ Estatística } \\
\cline { 2 - 7 } & $\mathbf{n}$ & $\mathbf{\%}$ & $\mathbf{n}$ & $\mathbf{\%}$ & $\chi^{\mathbf{2}}$ & $\mathbf{p}$ \\
\hline Sexo & 57 & 20 & 228 & 80 & & \\
Masculino & 6 & 10,5 & 73 & 32 & 10,826 & $0,001^{*}$ \\
Feminino & 51 & 89,5 & 155 & 68 & & \\
Estado civil & 57 & 20 & 228 & 80 & & \\
Solteiro & 5 & 8,8 & 17 & 7,6 & & \\
Casado/amasiado & 37 & 64,9 & 161 & 70,7 & 0,982 & 0,912 \\
Viúvo & 9 & 15,8 & 30 & 13,3 & & \\
Divorciado & 6 & 10,5 & 19 & 8,4 & & \\
Grau de instrução & 57 & 20 & 228 & 80 & & \\
Ensino fundamental & 49 & 86 & 171 & 75,1 & & \\
Ensino médio & 8 & 14 & 44 & 19,1 & 3,834 & 0,280 \\
Ensino superior & - & 0 & 8 & 3,6 & & \\
Analfabeto & - & 0 & 5 & 2,2 & & \\
Tabagismo & 57 & 20 & 228 & 80 & & \\
Sim & 4 & 7 & 8 & 3,5 & 1,392 & 0,238 \\
Não & 53 & 93 & 220 & 96,5 & & \\
Etilismo & 57 & 20 & 228 & 80 & & \\
Sim & 2 & 3,5 & 17 & 7,5 & 1,142 & 0,285 \\
Não & 55 & 96,5 & 211 & 92,5 & & \\
Freq. atividade física & 57 & 20 & 228 & 80 & & \\
Regular & 22 & 38,6 & 131 & 57,6 & & \\
Irregular & 13 & 22,8 & 29 & 12,7 & 8,669 & $0,013 *$ \\
Ausente & 22 & 38,6 & 68 & 29,8 & & \\
\hline
\end{tabular}

$\mathrm{GC}=$ grupo-controle; $\mathrm{GE}=$ grupo-estudo.

* Teste qui-quadrado. 
Foram encontrados, portanto, 57 pacientes que preencheram os critérios de TDM segundo a SCID-I/P 2.0 , representando uma prevalência de $20 \%$ de pacientes com TDM entre os matriculados na LHA.

A média de idade no GE foi de 52,5 $\pm 8,3$ anos, enquanto que, no GC, foi de 55,8 $\pm 9,5$ anos, observando-se diferença significativa entre os grupos $(\mathrm{p}=0,013)$.

Observa-se, também, diferença significativa na variável sexo entre o GE e o GC $(p=0,001)$, sendo que a razão de risco para depressão maior foi de 2,39 para mulheres, em relação aos homens (Tabela 3 ).
Os dados sociodemográficos relativos a estado civil, escolaridade, tabagismo e etilismo não mostraram diferença significativa entre GE e GC. Em relação à atividade física, verifica-se que os integrantes do GC apresentaram hábito de prática da atividade física regular com freqüência maior que os do GE, sendo essa diferença estatisticamente significativa $(\mathrm{p}=0,013)$, como pode ser observado na Tabela 3.

Ao serem comparados GE e GC no tocante às variáveis pressão arterial e bioquímica, observou-se diferença significativa quanto à pressão arterial diastólica $(\mathrm{PAD})(\mathrm{p}=0,040)$ e colesterol total $(\mathrm{p}=0,045)($ Tabela 4$)$

Tabela 4 - Comparação dos parâmetros avaliados entre GE e GC

\begin{tabular}{lllll}
\hline \multirow{2}{*}{ Variável } & GE $(\mathbf{n}=\mathbf{5 7})$ & GC $(\mathbf{n}=\mathbf{2 2 8})$ & \multicolumn{2}{l}{ Estatística } \\
\cline { 2 - 5 } & Média $\pm \mathbf{D P}$ & Média $\pm \mathbf{D P}$ & $\mathbf{Z}$ & $\mathbf{p}$ \\
\hline PAS & $139,00 \pm 26,87$ & $134,21 \pm 20,84$ & 0,600 & 0,548 \\
PAD & $91,35 \pm 17,03$ & $85,66 \pm 11,85$ & 2,055 & $0,040^{*}$ \\
IMC & $29,02 \pm 6,42$ & $28,70 \pm 5,37$ & 0,241 & 0,809 \\
Colesterol & $219,64 \pm 48,38$ & $205,45 \pm 43,97$ & 2,006 & $0,045^{*}$ \\
Triglicérides & $171,64 \pm 90,86$ & $171,28 \pm 117,99$ & 0,449 & 0,654 \\
Glicemia & $106,87 \pm 21,10$ & $107,78 \pm 31,62$ & 0,512 & 0,608 \\
\hline
\end{tabular}

$\mathrm{GC}=$ grupo-controle; $\mathrm{GE}=$ grupo-estudo; PAS = pressão arterial sistólica; $\mathrm{PAD}=$ pressão arterial diastólica; IMC = índice de massa corpórea.

* Teste: Mann-Whitney.

Considerando apenas os pacientes do sexo masculino, essa diferença foi mantida quanto à PAD ( $\mathrm{p}$ $=0,019$ ).

A SCID-I/P 2.0 fez a classificação dos seguintes diagnósticos, nos 57 (20\%) pacientes portadores de TDM: $16(28,1 \%)$ pacientes com TDM melancólico único; 24 (42,1\%) pacientes com TDM melancólico recorrente; seis $(10,5 \%)$ pacientes TDM atípico único; e $11(19,3 \%)$ pacientes com TDM atípico recorrente. Não foram realizados diagnósticos de depressão pósparto ou com sintomas catatônicos.

Entre os pacientes avaliados, nenhum se encontrava em tratamento prévio com fármacos antidepressivos.

\section{Discussão}

Há poucos estudos ${ }^{10-12}$ sobre prevalência de depressão entre pessoas com HA, sendo que as prevalências encontradas variam e podem depender da amostra e das escalas utilizadas.

Dilsaver \& Coffman ${ }^{11}$, comentando pesquisas realizadas entre 1959 e 1983 com a utilização de escalas e questionários (não especificados), referem sintomas depressivos importantes em torno de $37 \%$ de hipertensos, chamando atenção para o fato de que nenhum estudo foi realizado antes do início do tratamento anti-hipertensivo e que portadores de depressão atípica, transtornos do ajustamento com sintomas depressivos, distimia e TDM foram todos considerados como portadores de TD.

Fuller $^{12}$ se refere a taxas encontradas por outros autores variando entre 30 e $67 \%$ de sintomas depressivos entre pacientes hipertensos. Destaca, naqueles estudos, o uso de metodologias diferentes e diferentes definições de depressão e HA e, em sua pesquisa, encontra taxa de $28 \%$ de deprimidos hipertensos, usando como método diagnóstico os critérios constantes no DSM-III.

Simonsick et al. ${ }^{10}$, em amostra multicêntrica de hipertensos acompanhados por 10 anos, utilizando a escala Center for Epidemiologic Studies - Depression (CES-D), encontraram prevalência de depressão entre 9,4 e $13,5 \%$ para homens e 20,6 e $27,1 \%$ para mulheres, estabelecendo uma média de prevalência de $18 \%$, o que designou como alta sintomatologia depressiva, o que indicaria a possibilidade de serem pacientes caracterizados como portadores de TDM. 
indicaria a possibilidade de serem pacientes caracterizados como portadores de TDM.

A prevalência de $20 \%$ de TDM encontrada após a aplicação da SCID-I/P 2.0 é mais próxima do estudo de Simonsick et al. ${ }^{10}$ e parece refletir a realidade da nossa população. Embora a prevalência encontrada neste estudo possa parecer subestimada em relação à prevalência observada em outras pesquisas, deve ser considerado que aqueles autores trabalharam com escalas que definiam apenas sintomas depressivos e não especificamente diagnóstico de TDM, permitindo inferir que a variação desses resultados citados na literatura pode ser devida às diferentes formas de avaliação ${ }^{10-12}$.

Uma limitação dessa investigação pode ser o fato de não se ter aplicado a SCID-I/P 2.0 naqueles pacientes que apresentaram pontuação no BDI entre 10 e 15. No entanto, o estudo de Sprinkle et al. ${ }^{30}$, estabelecendo ponto de corte de 17 no BDI para associação com depressão maior, possibilitou o estabelecimento desse ponto de corte em nosso estudo.

Ao ser utilizado o BDI para a identificação dos pacientes deprimidos, excluindo aqueles classificados como depressão leve e fazendo a confirmação de depressão maior através de uma entrevista estruturada, tornou-se o diagnóstico mais preciso, evitando-se superestimação da prevalência na amostra aqui estudada.

Ao tomar-se, por exemplo, apenas os resultados do BDI para o ponto de corte acima de 15 , sem aplicação da SCID-I/P 2.0, seria encontrada uma prevalência de 27,7\% de sintomas depressivos, próxima aos resultados encontrados por Fuller ${ }^{12}$.

Mais ainda, considerando para o cálculo estatístico a amostra com pontuação ainda menor (entre 10 e 15 no BDI - "depressão leve"), seriam acrescidos mais 48 pacientes com o diagnóstico de TD sem especificação, o que elevaria a prevalência para $44,6 \%$, mais próxima, em média, aos resultados de Dilsaver \& Conffman ${ }^{11}$ e de outros autores citados por Fuller ${ }^{12}$.

A média de idade em nossa investigação, neste estudo, foi significativamente menor $(p=0,013)$ para os pacientes do GE, em comparação com aqueles do GC, o que contrasta com alguns autores ${ }^{35,36}$, mas está em consonância com os estudos de Scherrer et al. ${ }^{1}$ e Karpansalo et al ${ }^{37}$ com população masculina de meia-idade.

Com relação ao sexo, feita a correção para a proporção homens/mulheres presentes na LHA ( $1 / 3$ de homens e $2 / 3$ de mulheres), encontrou-se, ainda, uma prevalência de TDM 2,39 vezes maior para mulheres que para homens, número este discretamente superior ao relatado na literatura.

Os achados indicam, ainda, que os pacientes do GC realizam atividade física sistemática com maior freqüência que pacientes do GE $(p=0,013)$. É importante ressaltar que a atividade física é componente obrigatório tanto do tratamento de TDM quanto de $\mathrm{HA}^{7,9,21}$, e esse fato pode contribuir para diferenças importantes no resultado terapêutico.

Os dados encontrados relativos aos níveis de PA mostram valores significativamente mais elevados no GE para a PAD $(p=0,040)$, sendo observada essa mesma tendência com relação à PAS. Mesmo existindo diferença significativa apenas para a PAD, esse fato pode representar risco adicional para o aparelho cardiovascular.

Em relação a parâmetros bioquímicos (colesterol, triglicérides e glicemia), Shizuka \& Yambe ${ }^{38}$ encontraram dados de significância para aumento de colesterol total entre mulheres, embora refiram também essa tendência para os homens. Também em nosso estudo, encontramos diferença significativa relativa ao colesterol total $(\mathrm{p}=$ 0,045), quando comparados o GE e o GC (Tabela 4); e, embora não exista significância estatística quando separamos os grupos por sexo, observa-se uma tendência para níveis mais altos de colesterolemia entre as mulheres.

\section{Conclusão}

Foi encontrada uma prevalência de TDM em pacientes hipertensos superior àquela encontrada na população geral, além de dados consistentes quanto a maior nível de $\mathrm{PAD}$, colesterolemia e menor realização de atividade física entre indivíduos do GE.

Essa presença significativa de TDM e mesmo de sintomas depressivos não avaliados neste grupo de pacientes é fato preocupante, principalmente ao se levar em conta estarem ambas entre as doenças crônicas nãotransmissíveis mais freqüentes.

Deve-se destacar a necessidade de maior atenção, por parte dos profissionais de saúde, às queixas apresentadas pelo paciente hipertenso no que diz respeito a sintomas depressivos em atendimento primário, ambulatório específico e hospital geral, visando a um diagnóstico mais preciso e precoce e uma terapêutica mais eficaz.

Sugere-se a realização de estudos prospectivos no sentido de avaliar o impacto do tratamento do TDM para melhora do prognóstico e qualidade de vida de pacientes portadores de HA.

\section{Referências}

1. Scherrer JF, Xian H, Bucholz KK, Eisen SA, Lyons MJ, Goldberg J, et al. A twin study of depression symptoms, hypertension, and heart disease in middle-aged men. Psychosom Med. 2003;65(4):548-57.

2. Frasure-Smith N, Lespérance F. Depression and other psychological risks following myocardial infarction. Arch Gen Psychiatry. 
$2003 ; 60(6): 627-36$

3. Scalco AZ, Scalco MZ, Azul JB, Lotufo Neto F. Hypertension and depression. Clinics. 2005;60(3):241-50.

4. Carney RM, Freedland KE, Stein PK, Watkins LL, Catellier D, Jaffe AS, et al. Effects of depression on QT interval variability after myocardial infarction. Psychosom Med. 2003:65(2):177-80.

5. Wassertheil-Smoller S, Shumaker S, Ockene J, Talavera GA, Greenland P, Cochrane B, et al. Depression and cardiovascular sequelae in postmenopausal women. The Women's Health Initiative (WHI). Arch Intern Med. 2004;164(3):289-98.

6. Yan LL, Liu K, Matthews KA, Daviglus ML, Ferguson TF, Kiefe CI. Psychosocial factors and risk of hypertension; the coronary artery risk development in young adults. JAMA. 2003;290(16):2138-48.

7. Reiff M, Schwartz S, Northridge M. Relationship of depressive symptoms to hypertension in a Household//survey in Harlem. Psychosom Med. 2001;63(5):711-21.

8. Amaral GF, Porto CC, Brasil MAA, Jardim PCBV. Depressão e doenças cardiovasculares: importância para o clínico. Rev Soc Bra Clin Med. 2005;3(4):102-12.

9. Shinn EH, Poston WS, Kimball KT, St. Jeor ST, Foreyt JP. Blood pressure and symptoms of depression and anxiety: a prospective study. AJH. 2001;14(7Pt.1):660-4.

10. Simonsick EM, Wallace RB, Blazer DG, Berkman LF. Depressive symptomatology and hypertension-associated morbidity and mortality in older adults. Psychosom Med. 1995;57(5):427-35.

11. Dilsaver SC, Coffman JA. Hypertension and depression: question of a causal relationship persist. Psychiatry Res. 1988; 26(1):115-7.

12. Fuller BF. DSM-III depression and hypertension in two psychiatric outpatient populations. Psychossomatics. 1988;29(4):417-23

13. Abas M, Hotopf M, Prince M. Depression and mortality in a highrisk population. 11-Year follow-up of the Medical Research Council Elderly Hypertension Trial. Br J Psychiatry. 2002;181:123-8.

14. Glass R. Awareness about depression: important for all physicians. JAMA. 2003;289(23):3169-70.

15. Meredith LS. Depression: 20 years of progress. Med Care. 2004;42(6):499-501.

16. Mann JJ. The medical management of depression. N Engl J Méd. 2005;353(17):1819-34.

17. Lima MS. Epidemiologia e impacto social. Rev Bras Psiquiatr. 1999;21(SI):1-5.

18. Meyer CM, Armenian HK, Eaton WW, Ford DE. Incident hypertension associated with depression in the Baltimore Epidemiologic Catchment area follow-up study. J Affect Dis. 2004;83(2-3):127-33.

19. Markovitz J, Matthews KA, Kannel WB, D'Agostino RB. Psychological predictors of hypertension in the Framingham Study. JAMA. 1993;270(20):2439-43.

20. Jardim PCBV, Gondim MRP, Monego ET, Moreira HG, Vitorino PVO, Souza WKSB, et al. Hipertensão arterial e alguns fatores de risco em uma capital brasileira. Arq Bras Cardiol. $2007 ; 88(4): 452-7$.
21. Brasil, Ministério da Saúde. Controle da hipertensão arterial: uma proposta de integração ensino-serviço. Rio de Janeiro: CDCV/ NUTES; 1993. p. 232.

22. Jardim PCBV, Souza ALL, Monego ET, Barroso WKSS. Pressão arterial: semiotécnica e avaliação clínica do paciente. In: Porto CC. Doenças do coração: prevenção e tratamento. $2^{\mathrm{a}}$ ed. Rio de Janeiro: Guanabara Koogan; 2005. p. 504-9.

23. Sociedade Brasileira de Hipertensão, Sociedade Brasileira de Cardiologia, Sociedade Brasileira de Nefrologia. Diretrizes brasileiras de hipertensão arterial - IV DBHA. Rev Soc Bras Hipertensão. 2002;5:123-63.

24. Chobanian AV, Bakris GL, Black HR, Cushman WC, Green LA, Izzo JL Jr., et al. The seventh report of the joint national committee on prevention, detection, evaluation and treatment of high blood pressure (VII JOINT). JAMA. 2003;289:2560-72.

25. Cohen HW, Madhavan S, Alderman MH. History of treatment for depression: risk factor for myocardial infarction in Hypertensive patients. Psychosom Med. 2001;63(2):203-9.

26. Beck AT, Steer R. Beck depression inventory manual - II. San Antonio (TX): Psychological; 1993.

27. Gorenstein C, Andrade LHSG. Inventário de depressão de Beck: propriedades psicométricas da versão em português. In: Gorenstein C, Andrade LHSG, Zuardi AW, orgs. Escalas de avaliação clínica em psiquiatria e psicofarmacologia. São Paulo: Lemos; 2000. p. 89-95.

28. Cunha JA. Manual da versão em português das escalas Beck. São Paulo: Martins Fontes; 2001.

29. Spitzer RL, Williams JBW, Gibbon M, First MB. Users guide for the structured clinical interview for DSM-III-R. Washington, DC: American Psychiatric; 1990.

30. Sprinkle SD, Lurie D, Insko SL, Atkinson G, Jones GL, Logan AR, et al. Criterion validity, severity cut scores, and test-retest reliability of the beck depression inventory-II in a university counseling center sample. J Couns Psychol. 2002:49(3):381-5.

31. Tavares M. Entrevista clínica estruturada para o DSM-IV. Transtornos do eixo I: edição para pacientes - SCID - I/P (versão 2.0). Brasília DF; Universidade de Brasília; 1999.

32. American Psychiatric Association. Manual diagnóstico e estatístico de transtornos mentais (DSM-IV). $4^{\text {a }}$ ed. Porto Alegre; Artmed; 1995.

33. World Health Organization. Physical status: the use and interpretation of anthropometry. (Technical Report Series, 854). Geneva: WHO Report; 1995.

34. Monteiro Filho G. Segredos da estatística: pesquisa científica. Goiânia: Vieira; 2004.

35. Cervilla JA, Prince M, Joels S, Mann A. Does depression predict cognitive outcome 9 to 12 years late? Evidence from a prospective study of elderly hypertensives. Psychol Med. 2000;30(5):1017-23.

36. Bosworth HB, Bartash RM, Olsen MK, Steffens DC. The association of psychosocial factors and depression with hypertension among older adults. Int J Geriatr Psychiatry. 2003;18(12):1142-8.

37. Karpansalo M, Kauhanen J, Lakka TA, Manninen P, Kaplan G, Salonen JT. Depression and early retirement: prospective population based study in middle aged men. J Epidemiol Community Health. 2005:59(1):70-74.

38. Shizuka K, Yambe T. Relationship between depression and lipid metabolism in the elderly with hypertension. Jap J Geriat. $2001 ; 38(6): 785-90$. 